\title{
Beiträge zur Lehre von der Panophthalmitis.
}

\author{
Von \\ Dr. Schiess in Basel. \\ Hierza Taf. II. F'ig. 5 .
}

\begin{abstract}
Die Frage vom Zustandekommen der Panophthalmitis ist in letzter Zeit in diesen Blättern und anderwärts von mehr als einer Seite eingehender beleuchtet worden. Während Schweigger (d. A. VI. 2. S. 261 ff.) ein menschliches Auge mit Rücksicht hierauf zergliederte und den Antheil der verschiedenen Parthieen des Bulbus hierbei bestimmte, hat C. O. W e b er*) die entzündlichen Affectionen des Glaskörpers im Kaninchenauge mehr in's Auge gefasst und die normalen Gewebs-Elemente nachgewiesen, deren Derivate als trübe Streifen den Glaskörper durchziehen oder bei höhern Graden denselben vollständig in eine gelbe, trübe Masse verwandeln, und die er als wirklichen Eiter beschreibt.

Schweigger beschreibt in seinem Falle den Glaskörper als einen von feinen Membranen durchsetzten Eiterpropf und scheint dabei in Uebereinstimmung mit Weber an einen lokalen, entzündlichen Vorgang zu denken. Als neues Resultat wird dabei einer eitrigen Retinitis erwähnt.
\end{abstract}

V Virch ow Archiv XIX. S. 367 tf. 
Diesen Forschungen trat Ritter*) mit seinen experimentellen Untersuchungen am Kaninchen entgegen und entwickelte in einer ausführlichen und sehr gründlichen Weise seine Ansichten über Panophthalmitis, wobei er sowohl eine substantielle Betheiligung des Glaskörpers, als die Möglichkeit einer primären Retinitis in Frage stellt.

Bei diesem Stande der Frage möge es erlaubt sein, einen hierher gehörenden Fall in extenso mitzutheilen und einige allgemeine Erörterungen daran zu knüpfen.

Bei einem erwachsenen, schwarzgefleckten Kaninchen wurde in der Mitte der Hornhaut ein kleiner Lappen gebildet und derselbe wieder mit der Scheere in mehrere kleinere Parthieen getheilt, wobei sich bald eine bedeutende, tiefgreifende Entzündung der Gesammtgebilde des Auges einstellte. Während die Iris sich in die Wunde eindrängte, blieb in der Mitte dieser durch Hornhautreste und vorliegende Gewebstheile der Iris gebildeten Vorwölbung eine nabelförmig eingezogene Stelle, in der ein Eiterpropf sass, der sich immer frisch erneute. Der Bulbus veränderte dabei seine Form; er wurde mehr konisch, kleiner als der andere und matsch. Auch in den conjunctivalen Parthieen stellte sich eine ödematöse Schwellung und bedeutende Hypersekretion ein. Die Section wurde an 29. Tage vorgenommen, der Bulbus in die gewöhnliche chromsaure Lösung gelegt und 18 Tage nachher geöffnet.

Ausser der oben erwähnten Form-, Grössenund Resistenz-Veränderung zeigte das Auge in Kürze folgendes Verhalten: Der ganze Bulbus stellt eigentlich nur noch eine, mit einem eitrigen Propfe erfüllte Blase dar, deren vordere aufge-

*) d. Archiv VIII. 1. pag. 30 u. ff. 
quollene und verdickte Ränder aus verändertem, mit Pigment durchschwemmten Hornhautgewebe und Iris bestehend, zwischen sich eine Lücke lassen, die dem eitrigen Inhalt erlaubt, sich nach aussen zu ergiessen, bis die Afterproduktion der Binnengebilde sich erschöpft und bis die bereits begonnene Schrumpfung ihr Endstadium in jenem Zustande erreicht, den man als Phthisis bulbi wohl kennt. - Vom Linsensystem ist Nichts mehr zu erkennen, vom Glaskörper mikroskopisch ebenfalls Nichts mehr; die Sclera erscheint etwas verdickt, die Choroidea geht ohne bestimmten Absatz in die nach aussen an die Cornea gedrängte, hochgradig veränderte Iris über, so dass also jene ganze Blase den eitrigen Inhalt in einer pigmentreichen, noch als Membran sich charakterisirenden Finfassung zeigt. Kurz, wir haben das vollendete Bild einer Panophthalmitis.

Meine Absicht ist nicht die, nun der Reihe nach die Veränderungen der verschiedenen Membranen bei dem Prozess durchzugehen; ich möchte nur das Verhalten der Choroidea speciell hier in's Auge fassen und dabei einige Seitenblicke auf die Stelle der Retina und des Glaskörpers werfen. Ich wende mich zuerst zum Verhalten der äussersten Schichten der Choroidea, die man auch als lamina fusca bezeichnet. - Ich finde diese Schicht an "einzelnen Stellen etwas inniger, als normal, mit der Sclera verwachsen, ohne dass es dabei irgendwo unmöglich wäre, die Grenzen von Choroidea und Sclera scharf anzugeben. Von einer Durchsprengung der inneren Scleraschichten mit Pigment, wie es bei den ektatischen Veränderungen der Choroidea vorkommt, ist keine Spur vorhanden. Je weiter wir nach vorn gehen, desto intimer erscheint die Anheftung der Choroidea, und in der Gegend des Processus ciliares, der sich durch eine 
andere Aniage des Epithels deutlich genug markirt, erscheint es wirklich schwer, eine genaue Grenze zwischen Sclera und Strahlenkörper zu ziehen. Es mag hier noch bemerkt werden, dass ein sehr dentliches Hervortreten der Bindegewebskörper in der Sclera und einzelne Stellen mit Kernwucherungen entschieden auf eine Betheiligung an den weiter innen liegenden Vorgängen hinweist, ohne dass ausser einer einzigen Stelle, da wo die Sclera anfängt in die Cornea ïberzugehen', in der Gegend des Schlemm'schen Kanals, diese Wucherung eine eigentliche Trübung des intercellulären Gewebes zu Stande brächte. An jener lokalen Stelle freilich tritt nicht nur eine. Trübung der Intercellularsubstanz, sondern eigentliche Eiterbildung auf. Da in den äusseren, lockeren Lagen der lamina fusca die gleichen Vorgänge sich beurkunden, nur in geringerem Grade, als in dem eigentlichen Stroma der Choroidea, so mag füglich zur Betrachtung des eigentlichen Choroidealstromas geschritten werden.

Was zuerst das eigentliche Stroma der Choroidea anbetrifft, mit dem die lamina fusca ein ähnliches Verhalten zeigt, so bemerkt man auf senkrecht geführten Schnitten zunächst eine bedeutende Verdickung desselben, und zwar sind die Interstitien der pigmentirten Stromazellen besonders gewachsen. -- Während sonst Pigmentzelle an Pigmentzelle sich reiht, sieht man die Züge der pigmentirten Zellen hier durch helle, pigmentlose Schichten von verschiedener Mächtigkeit getrennt (s. Fig. 5 Taf. II.), ohne dass in der Lagerung gerade cine bestimmte Anordnung herrschte. Bei näherer Betrachtung sehen wir diese pigmentlosen Schichten aus einem feinen Netzwerk bestehen, mit vielen kleinen Zellen oder Zellenkernen. Es sind runde Körper ohne deutliche Abgrenzung von Zellenmembran und Inhalt, von einem mittleren Durchmesser von $0,0035 \mathrm{~mm}$. Häufig sieht man spindelförmige Gebilde, in denen ein orler murere solcher Kerne sitzen 
und an truberen Stellen schreitet die Kernewucherung in der Weise fort, dass in einer solchen spindelförmigen Zelle eine ganze Reihe von Kernen hinter einander liegen (s. Fig. 5 c. Taf. II.). Ich kann mich daher hier mit Ritter vollständig einig erklären, der die Entstehung des Eiters aus den pigmentlosen Stromazellen ableitet, nur scheint hier die Wucherung eine weitergehende gewesen zu sein, indem an sehr vielen Stellen die Ableitung einer Mehrzahl von Kernen oder jungen Eiterkörperchen aus einer präformirten Zelle evident ist. In den äusseren Schichten erscheinen dabei die Kerne noch mehr länglich und man sieht nur sparsam freie Kerne; das trübere Faserwerk des Bindegewebes tritt noch mehr in's Auge, während, je weiter wir nach innen gehen, desto mehr die freien Eiterkörper in den Vordergrund treten. Dass hier auch die Kerne bereits grösser werden und das eben angeführte Maass zuweilen um das Doppelte übertreffen, kann ich ebenfalls mit Ritter bestätigen.

In den Stromazellen kann ich keine besonders auffälligen Veränderungen wahrnehmen, als dass ihre Ausläufer an einzelnen Orten sich weniger scharf abgrenzen, doch ist auch das nicht immer der Fall; man sieht zum Beispiel einzelne ganz normale Stromazellen in die Massen der Choriocapillaris hineinragen, ohne dass sie sich un das rege Zellenleben rings her irgendwie zu bekümmern scheinen. Andere Parthien freilich scheinen denn doch gelitten zu haben, aber nicht etwa auf dem Wege der entzündlichen Vorgänge, sondern wahrscheinlich durch den Druck und die mangelnde Ernährung.

Auffällig ist besonders der Mangel an Gefässen. Während ja im normalen Zustande die Choroidea wesentlich beinahe als ein Gefässnetz kann bezeichnet werden, scheint hier unter der zellulären Entwicklung der cirkulatorische Apparat in auffälliger Weise gelitten zu haben. Es kennzeichnet besonders dieser Befund unseren Fall 
als einen entschieden bereits jenseits der $\Lambda$ kme der Entzündung stehenden. In der Gegend vor der ora serrata werden auf senkrechten Durchschnitten Gefässdurchschnitte auch in den tiefen Schichten wieder häüfiger, während in den mehr äquatorialen Theilen beinahe nur in der Choriocapillaris solche zu finden sind. Schweigger giebt an, dass das Bindegewebe um die Gefässe der Ausgangspunkt der Fiterung sei, es ist das eine Frage, für deren Discussion unser Fall wenig geeignet erscheint. Ritter statuirt anfangs eine bedeutende Zunahme der Gefässvolumina, sagt, dass sich die Gefässe wahrscheinlich auch vermehren, eine Behauptung, beiläufig gesagt, die wohl vergleichende Injectionen an gesunden und kranken Augen erforderte, und die mir überhaupt bei einer so gefässreichen Haut, wie die Choroidea ist, schwer nachzuweisen scheint. Im Gegensatz zu Schweigger giebt Ritter an, dass die Zellen der sie begleitenden Bindegewebszüge sich erst spät vermehren und dass dies dann zu einem allmäligen Untergange der Gefässe fuihre. In unserem Falle möchte wohl der Druck der massenhaft wuchernden Stromazellen einen verhältnissmässig so hochgradigen Schwund der Gefässe herbeigeführt haben.

Flächenansichten der Choroiden lassen sich hier sehr schwer gewinnen; das Gewebe ist so brüchig geworden, so sehr von dem Eiter durchsetzt, dass nur kleinere Partikel der Choroidea mit anhaftenden Eitermassen sich ablösen lassen. Auch hier sind keine deutlichen Gefässe mehr zu erkennen, daher gerade auch die grosse Brüchigkeit, da keine grösseren Flächenausdehnungen gemeinschaftlicher Gewebseinheiten mehr existiren. Auffällig und bemerkenswerth ist besonders das stätige Wachsthum der Zellenwucherung von aussen nach innen. (s. Fig. 5 Tafel II.)

Wenden wir uns zur Choriocapillaris, als der innersten Schicht, so findet sich auch in unserem Falle, 
entsprechend den Darlegungen von Schweigger und Ritter, an die sich hier auch Heymann*) anschliesst, der wesentlichste Heerd der Veränderungen gerade hier. Wie schon oben angeführt, hat überhaupt die Choroidea bedeutend an Dicke zugenommen, an einzelnen Stellen wird sie bis $0,50 \mathrm{~mm}$. dick, wobei denn stark die Hälfte auf die Choriocapillaris mit Stratum pigmenti fällt. Es stellt diese Schicht im Allgemeinen eine nach aussen von den Stromazellen der Choroidea, nach vorn von dem Pigmentepithel ziemlich parallel begrenzte, gelbliche, körnige Schicht dar, in der sowohl einzelne, dicht gedrängte Bindegewebsfasern, als auch hie und da Quer- und Längsschnitte von Gefässen sich erkennen lassen (s. Fig. 5 Taf. II.). Die Zellenproliferation ist eine enorme, man sieht die Zellen zum Theil Reihen bilden mit anderen etwas weiter nach aussen gelegenen; hie und da ragen auch Stromapiginentzellen in mehr oder weniger regelmässigen Formen in die innerste, gelbe Schicht hinein, ohne aber ihre Continuität aufzuheben Es lässt sich vielmehr diese Schicht auf senkrechten Schnitten in continuo vom Eintritt des opticus bis gegen die Gegend der processus ciliares verfolgen.

Richten wir nun unsere Blicke auf die innere Begrenzung dieses Eiterlagers, so stossen wir hier auf das Pigmentepithel. An einzelnen Stellen lässt sich dessen Anordnung als eine verhältnissmässig noch normale erkennen, doch sind dies Ausnahmen. - Schon mit blossem Auge sieht man vor der oben erwähnten eiterdurchdrungenen Choriocapillaris eine schwarze Contour, sie von dem centralen Eiterhaufen abgrenzend. Oft ist es nur eine einzige Zellenreihe, welche die Umsäumung der Choriocapillaris übernimmt, sehr gewöhnlich treten aber verschiedene Unregelmässigkeiten ein; es erscheinen ein-

*) Archir f. Ophthalm. VII. 1. pag. 133. 
zelne Zellen mehr gegen einander oder über einander geschoben; sie verlieren ihre normale Form; die Zellen werden mehr länglich; man sieht neben Gebilden, die durch Kern und Inhalt sich noch als Pigmentepithel kennzeichnen, mehr rundliche, eckige Pigmentkörper, die aber immer noch ein gewisses Volumen beibehalten und sich zu unregelmässigen Pigmentconglomeraten ballen. Daneben sind Lokalitäten, wo eine Wucherung des Epithels vermuthet werden könnte, obwohl auch hier noch der Ausweg offen bleibt, an eine massenhaftere Zusammenschiebung der Pigmentepithelien von der Seite her zu denken. In der Gegend der ora serrata, wo der Zusammenhang des Epithels ein innigerer und derberer zu sein scheint, finden wir eine eigenthümliche Gestaltveränderung. Während an andern Stellen der Strom des Eiters die Zellen auseinander getrieben, hat er hier zwar auch die Schicht derselben in einzelne spitze Buchten gegen das Innere des Auges vorgedrängt, so dass ihre Contour eine spitze Zickzacklinie darstellt, so dass also die Spitze jedesmal der Stelle der höchsten Druckwirkung entspricht. An anderen Theilen fruilich, und zwar sind es besonders die äquatorialen Parthieen, ist der Andrang der von hinten und aussen kommenden neugebildeten Massen so gewaltig gewesen, dass er die hemmende Schranke des Epithels zerbrach. An anderen Stellen fand nur eine einfache seitliche Verschiebung statt und in die dadurch entstandenen Lücken keilten sich die jungen Eiterkörper ein. Je nach der grösseren oder geringeren Gewalt nun ist das Epithel auch wieder verschieden gelagert; an einzelnen Stellen sehen wir es in kleine Partikel getheilt, mit in den Glaskörperraum hineingeschwemmt, so dass es gar nicht mehr möglich ist, seine ursprüngliche Lagerung zu definiren, anderwärts bleiben die Hauptmassen an der ursprünglichen Stelle und lassen nur kleine Parthieen nach innen dringen, die dann, je nach dem lokalen 
Verhalten der Retiva, letatere in ihren äusseren Theilen imprägniren oder aber in den Glaskörperraum vordringen. Eigenthümlich ist die Anordnung der abgelösten und fortgeschweminten Pigmentepithelien; sie liegen alle in der Richtung eines Stroms gelagert, der von der Choroidea aus nach innen und zugleich nach vorn gegen jenen mittleren offenen Kanal streicht, der zwischen den Irisrudimenten und Hornhautresten offen geblieben resp. durch ein Coagulum von Eiter ausgestopft ist. Man sieht dies besonders deutlich an jenen Stellen, wo die Hauptmasse des Epithels am ursprünglichen Standort sitzen geblieben und ihre Ausläufer mehr nach innen liegen. Diese Stromrichtung ist übrigens auch an den ausgewaschenen Netzhautresten zu verfolgen.

Kerntheilungen des Epithels konnte ich nirgends finden. Die vollständigste Zerstreuung des Pigments entspricht aber jenen Stellen, wo die Retina ganz zerstört und wo nur noch dunklere, grosskörnige Gruppen ihre frühere Existenz ahnen lassen.

Die Glashaut der Choroidea kann ich nirgends mehr erkennen.

Die Retina bietet in unserem Auge, je nach der lokalen Intensität der Eiterproduction und der serösen Ausschwitzung ein ziemlich verschiedenes Verhalten.

Wir finden Stellen, wo noch sehr deutliche Retinatheile auf dem Pigment aufsitzen, freilich keine einzige, wo normale Retinastruktur irgend beibehalten, ja nur noch die bekannte Schichteneintheilung zu constatiren wäre. Verhältnissmässig am besten erhalten zeigt sich die Netzhaut an Stellen, wo über ihr noch Glaskörper erhalten ist.

In der Gegend der papilla nervi optici scheint ein besonders reger Proliferationsprozess stattgefunden zu haben; die Opticusbündel scheinen ziemlich in der Mitte auseinander gedrängt, so dass ein mit Eiterkörperchen 
gefüllter Trichter entsteht, dessen Spitze bis hinter die lamina cribrosa zurückreicht. An der Unbiegungsstelle von der Papille in die Retina scheinen die Opticusfasern abgerissen und gehen gerade nach vorn gegen die Mitte des Auges, überall ausgefasert und mit eitrigen Bestandtheilen durchsetzt. (s. Fig. 5 f. Taf. II.)

Das gesammte Netzhautgewebe erscheint sehr trübe; auch nach Zusatz von Natron bleibt es viel dunkler, als die umliegenden eitrigen Massen und als die hinterliegende Aderhaut. Hie und da bezeichnen nur trübe, fasrige oder körnige Haufen, auf der Choroidea aufsitzend, das ehemalige Netzhautgewebe. Betrachten wir diejenigen Stellen, wo die Retina noch auf der Choroidea aufsitzt oder wo nur ein kleiner Erguss, der inselförmig vor dem Pigmentepithel unter der Netzhaut sitzt, dieselbe an ganz circumscripter Stelle abgelöst, so sehen wir hier Durchdringung der Retina-Elemente mit eitrigen Produkten. Serös durchtränkte Nervenfasern, Radiärfasern mit ziemlich grossen, hie und da zahlreich werdenden blassen Kernen, einzelne Ganglienkugeln und Elemente der Körnerschicht sind die zurückgebliebenen Rudera der Netzhaut.

Die Radiärfasern erscheinen durchgehends breit, aufgequollen, haben eigentlich weniger mehr eine fasrige Strultur, sind vielmehr reiche, breite Bündel; an einzelnen Stellen sind die Körner noch deutlich vorhanden, an anderen sind sie verschwunden und die Bündel erscheinen nakt, wie macerirt und ausgewaschen oder ausgeschwemmt. Durch alle Elemente hindurch geht cine ganz feine, erst bei stärkerer Vergrösserung mehr hervortretende Tüpfelung, wie sie bereits Ritter beschrieben und abgebildet hat. - Die Begrenzung gegen die Choroidea hin erscheint durchgehends noch distincter, als die gegen den Glaskörperraum. Hier fasern sich nämlich die Retina-Elemente ganz unregelmässig aus: einzelne Fragmente sind in den Glaskörperraum hineingeschwemmt und verlieren sich in 
dem allgemeinen Chaos, das aus Retina, Glaskörper, Choroidea und Eiter zusammengesetzt ist. Von der limitans sehe ich nirgends Reste, treffe dabei auch manche Stellen, wo von der Retina überhaupt keine Spur mehr zu finden, wo dagegen der Eiter ungehindert zwischen einzelne Pigmentreste in den inueren Augenraum sich ergiesst. Die Hauptmasse des mittleren Propfes besteht aber aus Eiter in einer fasrigen Grundsubstanz, die übrigens in den mehr centralen Theilen sehr zurücktritt.

Vom Glaskörper sind nur spärliche Reste übrig geblieben, besouders in den hinteren Parthieen in der Nähe des Opticuseintritts. Fr stellt hier hellere Stellen dar, die ein feinfasriges Gerüste bilden, mit einzelnen eingestreuten, runden Kernen und grösseren Zellen mit grossen Kernen; doch ist diese Zellenbildung gar keine reichliche.

Die Gegend des processus ciliares zeichnet sich hauptsächlich nur durch eine stärkere Anhäufung von Pigment aus, und zwar sowohl des Stromapigments als des Pigmentepithels; ersteres erscheint mehr in einander verfilzt, als weiter hinten, so dass hier keine solche pigmentlosen neugebildeten Schichten sich nachweisen lassen. Von muskulösen Gebilden lässt sich nichts mehr wahrnehmen. Dickere, trübere Massen, die gerade entsprechend dem Strahlenkörperkranz in dem Innern des Auges liegen, scheinen diese Gegend als cinen Hauptfaktor bei dem panophthalmitischen Prozess zu kennzeichnen.

Vom Linsensystem findet sich auch keine Spur mehr vor.

Auf das Verhalten der Iris gehe ich nicht näher ein; ihr Gewebe ist ebenfalls sehr hochgradig verändert; ihr Pigment zum Theil aus den Zellen getreten, zum Theil in die anliegende Hornhaut, zum Theil in die mittlere Ausfüllmasse geschwemmt; ihr aufgequollenes Gewebe in unmittelbarer Continuität mit den exsudativen Massen. 
Die von hinten nachrückenden Masseu bedingten den schon früher erwähnten Durchpass zwischen ihren aufgelockerten Rändern.

Nach dieser eingehenden Beschreibung des vorliegenden Falles möge es mir erlaubt sein, einige allgemeine Beobachtungen hier anzuknüpfen. - Zuerst zur Krankheitsgeschichte des vorliegenden Falles. - Ich denke mir ungefähr folgende Reihenfolge der Krankheits-Erscheinungen.

Nach Eröffnung der Cornea, wobei auch die Kapsel gesprengt wurde, lagerte sich die Iris gegen die Hornhaut; die Linse trübte sich rasch, quoll auf und wurde nach und nach vollständig entleert; ihre Quellung diente als Fntzündungsreiz für die tieferen Organe, dabei mag auch direkt von der Iris aus der Reiz auf die Aderhaut fortgepflanzt worden sein. Hatte einmal die massenhafte Fiterproduction in der Choroidea begonnen, so ist auch die Netzhautablösung und Erfüllung des Augeninnern mit der erwähnten eitrigen Masse erklärt.

Vergleiche ich den Fall mit denjenigen, die Ritter*) beschrieben, so finde ich an meisten Aehnlichkeit mit Fall VI., wo 25 lage nach der Verletzung exstirpirt wurde, während ich 29 'Tage nach derselben den Bulbus entfernte. -... Ls ist jedoch die Veränderung im Innerı des Auges in meinem Falle eine viel weiter gehende, die Vorbereitung zum Ausgangsstadium der Panophthalmitis, der Phthisis bulbi eine viel weiter vorgeschrittene. Bei Ritter scheint die Retina in ihrer Continuität nirgends. aufgehoben; Choroidea und Retina sind vielmehr untrennbar verwachsen, während hier die enorme Zellenproduktion alle Organisation zertrümmert, das Pigmentepithel in den Glaskörperraum hineintührt und die Retina ibrer nervösen Elemente beraubt, bis nur das ausgewaschene, Archiv flir Ophthalmologie. IX. 1. 
mannigfach alterirte Gerüste der Radiärfasern zurückbleibt. Der entzündliche Prozess beginnt bereits sich zu erschöpfen; die regressiven Vorgänge, die sich schon durch das Matschwerden und die Formveränderung des Bulbus angekündet, sowie durch den Schwund rer Gefässe, würden bei längerem Leben des Thicres wohl mit Macht bald begonnen haben.

Dass wirklich ein Fall von Panophthalmitis der exquisitesten Art hier vorliege, wirl nach der vorausgegangenen Beschreibung wohl Niemand bezweifeln. Offe'lbar hat auch hicr, wie bei Ritter, der entzinndliche Prozess seinen Iauptsitz in der Choroidea und von da aus hat sich das gesetzte Produkt zum Theil in sehr stürmischer Weise in's Innere des Auges ergossen. Die Ausgangsgebilde der Eiterung sind die pigmentlosen Stromazellen. Man sieht dieselben sowohl auf sonkrechten Schnitten als auf Flächenansichten ganz ungewöhnlich zahlreich und zwar besonders in der innersten Aderhautschicht. Darin stimme ich mit Ritter vollständig überein, nur gcht dic Wucherung dieser Zcllen weiter, als in seinen Fällen; während er nicht mehr als zwei Kerne beobachtet, sehe ich hic und da ganze Reihon solcher rundlichen Gebilde hinter einander sitzen, bis es in der innersten Schicht iiberhaupt nicht mehr möglich ist, irgend cine Genese zu erkennen, da hier ein junges Fiterkörperchen unmittelbar am anderen liegt. Die Entwickelungsgeschichte des Fiters studirt sich besser an den Stellen, wo neben dem veränderten Gewebe noch normales oder doch wenig alterirtes liegt.

Auf Flächenansichten erblickt man zwischen den Stromapigmentzellen stärkere bindegewebige Züge und besonders viele elastische Fasern, die bogenförmig begränzte Nester von nougebildeten eitrigen Massen umschliessen. lis entsteht dadurch eine Art alveolärer Formationen Diese Ausbildung der elastischen Flemente der 
Aderhaut zu solchen alveolären Ausbuchtungen habe ich auch schon anderwärts beobachtet, z. B. bei lokalem Auftreten einer malignen Neubildung in der Choroidea, wo die neugebildeten Zellen ebenfalls in solche elastische Rahmen eingefasst waren - Anderwärts liegen die neugebildeten Körperchen in gleicher Schichtung mit dem Stromapigment. An einzelnen Stellen zeigt letzteres, wie schon beobachtet, eine etwas undentliche, verwischte Begrenzung, von einer Zellenwucherung in diesen Theilen findet sich auch keine Spur. Die Stromrichtung nach innen erklärt die geringe Betheiligung der Sclera.

Den Hauptbrennpunkt des ganzen Prozesses finden wir in der Choriocapillaris. Hier werden die meisten Eiterkörper gebildet und hierher drängen sich auch die Produlte der äusseren Schichten, zwischen den Stromapigmentzellen sich durchwindend, bis der Druck auf die resistente Pigmentephitelschicht ein so grosser geworden, dass ihre Reihen auseinander weichen. Mit der Zellenproduktion geht ohne Zweifol, wie anch Schweigger and Ritter angeben, eine Ausscheidung bedeutender Massen von einer fibriuhaltigen Flüssigkeit Hand in Hand, in der die zelligen Theile suspendirt sind; diese Flüssigkeit durchdringt dic vorhandenen Gewebstheile, lockert sie auf, macht sie permeabler und drängt die einzelnen Schichten auseinander. Diese seröse Durchtränkung, die bereits auf ein Circulationshemmniss hindeutet und die verschiedene deletäre Prozesse an Auge begleitet, auch als Chemosis der Conjunctiva uns von tieferen Prozessen äusserlich Kunde giebt, muss in unserem lialle eine zienulich hochgradige gewesen sein, darauf deutet die so deatliche Ausprägung einer gewissen Stromrichtung in der Stellung zum Beispiel der Pigmentepithels. Dass die Schicht der Epithels besonders in den vorderen Parthien eine bedeutende Resistenz bietet, mag man an jener zickzackförinigen Hervorwölbung derselben in der Gegend 
des processus ciliares ersehen. Dass dagegen auch ohne ergiebige Berstung des Epithels Eiter in ziemlichen Massen nach innen dringen kann, beweisen jene kleinen circumscripten Netzhautablösungen, hinter denen sich die Linie des Epithels ungestört fortsetzt.

Freilich begnügt sich der Eiter an den wenigsten Orten mit einer so engen Passage; die hemmenden Schranken werden durch den gewaltsam andringenden Strom durchbrochen, die Elemente des Epithels bei Seite geschoben, über einander gehäuft. Dabei leidet aber anch die Selbstständigkeit dieser Zellen; sie erleiden verschiedene Defigurationen, lösen sich auf und ihre Fragmente werden mit dem Eiterstrom bis gegen die Mitte des Bulbus hingeschwemmt. Es fängt dann an solchen Stellen die allgemeine Eitermasse unmittelbar über dem Stromapigment an, das dann öfters in einzelnen seiner Elemente in diese allgemeine Cloake hineinragt. Die zerstreuten Pigmentmoleküle deuten die Existenz eines früheren Epithels nur noch schwach an. Klebs hat unlängst (in Virchow's Archiv XXV. pag. 379) in einem phthisischen Bulbus, der wegen neuerdings auftretender entzündlicher Erscheinungen exstirpirt wurde, verschiedene Pigmentlagen vorgefunden, die er aus verschiedener Quelle herleitet, eine mehr centrale aus Blutfarbstoff nämlich, während er bei der äusseren es zweifelhaft lässt, ob der Blutfarbstoff oder das Choroidealpigment hier die Ursache der Pigmentirung sei. Dass eine solche Pigmentirung durch Blutfarbstoff möglich sei, stehe ich nicht an, zuzugeben; in unserem Falle lässt sich auch die diffuse Pigmentirung in der Mitte des Auges mit Sicherheit auf das Choroidealpigment zurückführen.

Die Netzhaut ist, wie schon oben bemerkt, hier in sehr bedeutendem Grade verändert. Während einzelne Parthieen noch an der Choroidea anlagern, von Eiterkörpern durchzogen, erscheinen andere Parthieen lokal abge- 
löst und wieder andere vollständig aus ihrem Zusammenhang getrennt und mit dem Eiter zu einer Masse verfilzt, nur durch eine etwas dunklere Färbung sich noch als Netzhautderivate kennzeichnend. Dies Verhalten deutet ebenfalls mit Evidenz auf einen von der Choroidea ausgehenden, nach innen tendirenden Entwickelungsstrom, der eben je nach seiner Mächtigkeit diese verschiedenen Wirkungen hervorruft. Zwischen und auf den aufgequollenen radiären Fasern sieht man Reihen von Eiterkörpern. Ausser diesen Körpern kommen auch noch blasse Kerne in den Radiärfasern vor, die niemlich gross und schwach granulirt sind: diese Granulation lässt sich übrigens durch die gesammten fasrigen Reste der Netzhaut verfolgen, wie auch Ritter bereits nachgewiesen hat. Bei der Beurtheilung von dem Verhalten der Retina beim panophthalmitischen Prozess stehen sich Schweigger und Ritter ziemlich diametral gegenüber. Während Schweigger eine eigentliche eitrige Retinitis annimmt, läugnet Ritter nicht nur die Möglichkeit einer Eiterproduktion in der Netzhant, sondern beanstandet die Retinitis überhaupt. In unserem Falle sehe ich eine Durchdringung des radiären Fasersystems mit eitrigen Körpern und Auftreten von vergrösserten Kernen, daneben eine Ausfaserung des Retinagerüstes mit Aufquellung. Ohne Zweifel wird eine Aufquellung der Retina in Folge der Exsudation der Chorioidea stattgefunden haben, dem folgte der Durchbruch des Fiters von der Chorioidea aus: nun wurde die Netzhaut entweder in kleinen Parthieen abgelöst oder aber das eitrige Produkt ergiesst sich durch die Netzhaut hindurch in den Glaskörperraum, wobei die Retina nach und nach in der Weise alterirt wurde, bis sie endlich dem Drucke nachgab und ihre Theile selber in der eitrigen Masse untergingen. Hierbei ging vorerst die Limitans verloren, die Körner lösten sich nach und nach ab, so dass auch an den Stellen, wo keine Ablösung 
oder Zertrümmerung stattfand, doch nur das ausgewaschene Radiärfasersystem übrig blieb. In den abgelösteu Nestern von Netzhautsubstanz lassen sich wohl noch Gebilde finden, die Körnern und Ganglien entsprechen, doch sind letatere nicht mohr häufig. - - Jedenfalls diufte in unserm Falle der Licht percipircnde Theil der Retina sehr früh functionsunfühig geworden sein.

Dass das erste Starlium, die blosse seröse Durchtränkung der Netzhaut und Choroidea, noch eine Restitutio ad integrum zulïsst, ist den Praktikern wohl bekannt; freilich dürfen im Algemeinen diese \%ustände nicht zu lange fortdauern.

In Schweigger's Falle hatte cine totale Netzhautablösung stattgefunden, ein Umstand, der dic Physiognomie des kindresultates in manchen Punkten alteriren kann. Mir scheint daher Ritter in Unrecht zu sein, wenn or a priori eine citrige Retinitis lengnet; es dürfte siberhaupt wohl der \%eityunlit noch nicht gekommen sein, wo es erlaubt wäre, eine abschliessende Systematik der pathologischen Vorgänge in Inneru des $\Lambda$ uges zu geben: auch sind menschliche Augen und Thieraugen doch nicht absolut zu parallelisiren, so erwünschte Aufschlüsse auch das verständig angewandte lixperiment giebt. Cnsere Aufgaben möchten noch cinige Zeit sein, möglichst erschöpfende Finzelbeobachtungen zu geben, und diese Hand in Hand mit der Beobachtung im lebenilen Auge werden langsam zwar aber sicher zu ciner crschöpfenden Kenntniss jener Vorgänge führen, deren Lirforschung seit $\mathrm{Helm}$ holtz's Entdeckung allerdings, man darf wohl sagen, höchst erfreuliche Resultate für Wissenschaft und Praxis geliefert.

So möchte ich denn über dic Frage gerade der eitrigen Retinitis, wie der Retinitis überhaupt, gerne die Akten noch offen behalten. Dass bei heftigen Reizen der Choroidea ihr Produkt vollstïndig ausreiche, um den 
Ganzen Bulbusrium in einen eitrigen Pfropf zu verwandeln. Dass eine solche Wanderung des Eiters von der Choroidea nach innen stattfindet, dass dadurch die Netzhaut zerstört, der Glaskörper verdrängt werden kann, geht allerdings aus den Beobachtungen von Ritter und mir hervor. Auch die Schwierigkeit, wie durch eine Wucherung der im normalen Zustand wenig beachteten pigmentlosen Stromazellen ein so massenhaftes Produkt gesetzt werden könne, scheint durch meine Beobachtung einer vielfachen Kerntheilung gehoben.

Dass die Eiterbildung in meinem Falle nicht etwa dem Glaskörper zur Last falle, ist ausser Zweifel schon durch das Verhalten der noch restirenden Theile des Glaskörpers und durch die unmittelbare Beobachtung des Eindringens der jungen Zellen in den inneren Raum des Auges. Eine Literbildung des Glaskörpers, wie sie W eb e r beschreibt, ist hier jedenfalls nicht vorgekommen. Wahrscheinlich war aber die Produktion der Choroidea gleich so rasch, dass der Glaskörper bald verdrängt wurde, und zwar denke ich mir, dass er mit den eitrigen Massen sich wach aussen entleerte. Dass im Glaskörpèr eine wirklich autochtone Fiterbildung stattfinden kann, kann ich vollkommen bestätigen, eben so gut als die Gefässneubildung. Selbst in unserem Falle finden sich, wie schon oben erwähut, in den freilich spärlichen Resten des Glaskörpers neugebildete Zellen, die allerdings nicht massenhaft auftreten und jedenfalls jeine wesentliche Rolle bei dem Prozesse gespielt.

Es scheint mir damit ein gewisser Typus für rasch sich entwickelnde Panophthalmitis traumatischer Ursache präcisirt; ich glaube aber zugleich, dass wir dies nicht als bindendes Paradigma vorerst aufstellen dürfen. Es giebt andere, mehr chronische Prozesse, bei denen unter dem Auftreten von Gefässneubildungen und Zellenwucherungen eine tiefere Veränderung der gesammten 
Gebilde des Bulbus vor sich geht, die man einstweilen wohl auch noch als entzündliche wird bezeichnen müssen. Allerdings versteht man gewöhnlich unter der Panophthalmitis einen stürmisch auftretenden Prozess und hier möchte ich mit Ritter annehmen, dass die Choroidea den grössten Theil der neuen Produkte liefert.

Basel, im Dezember 1862.

Dr. Schiess.

\section{Erklärung der Figur.}

Senkrechter Durchschnitt der Sclera, Choroidea und Retina bei 100facher Vergrösserung aus einer äquatorialen Parthie.

a. Sclera.

b. Pigmentstromazellen.

c. Sehichten der wuchernden pigmentlosen Stromazellen.

d. Choriocapillaris mit einzelnen breitgedrückten Gefässdurchschnitten.

e. Durchbrochene, unregelmässige Schicht des Pigmentepithels.

f. Mit eitrigen Reihen durchsetzte Retina. 
Fig. 4.

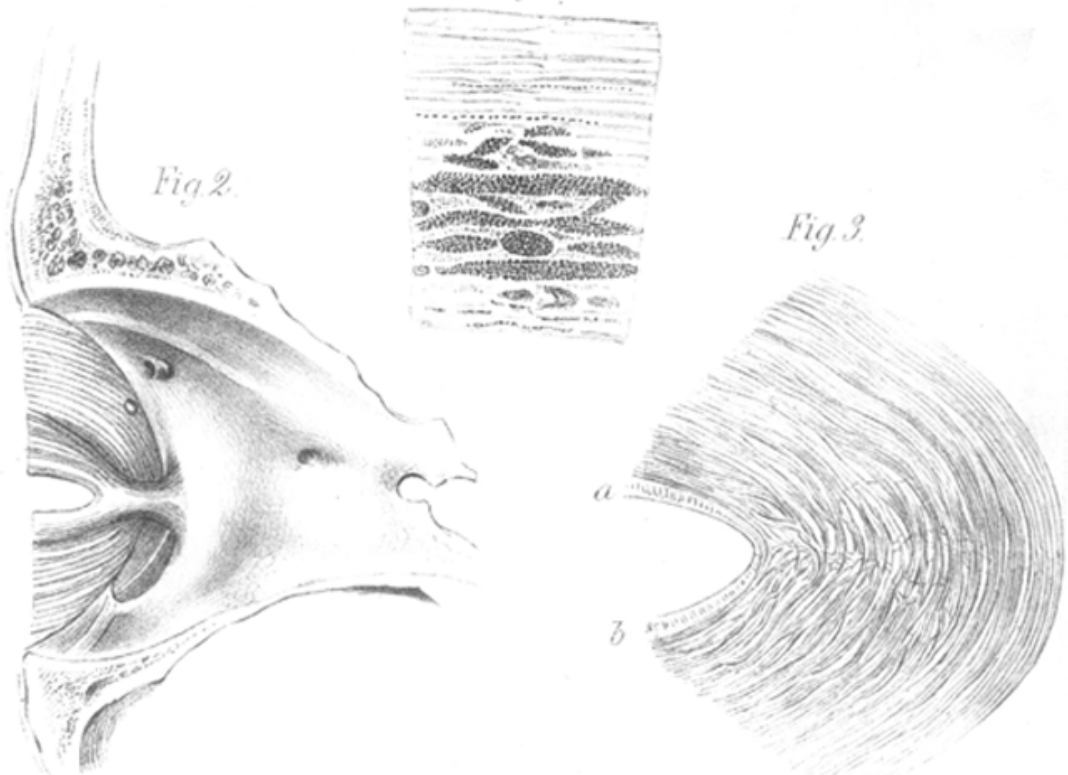

Fig 5

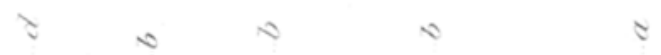

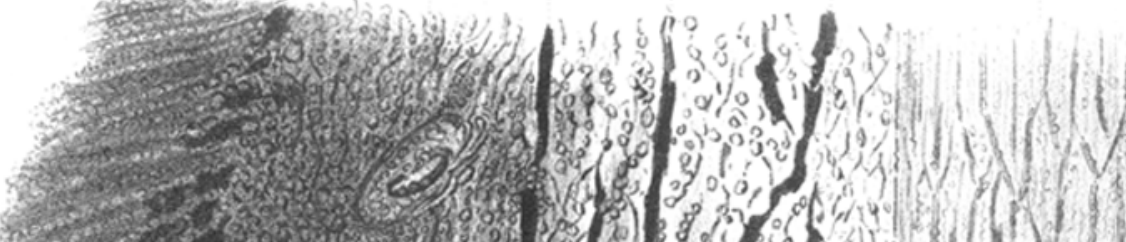

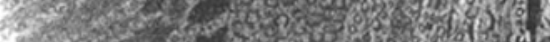

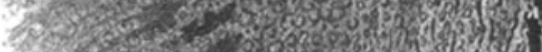

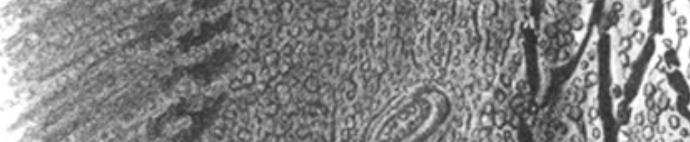

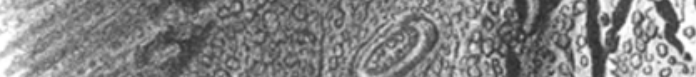

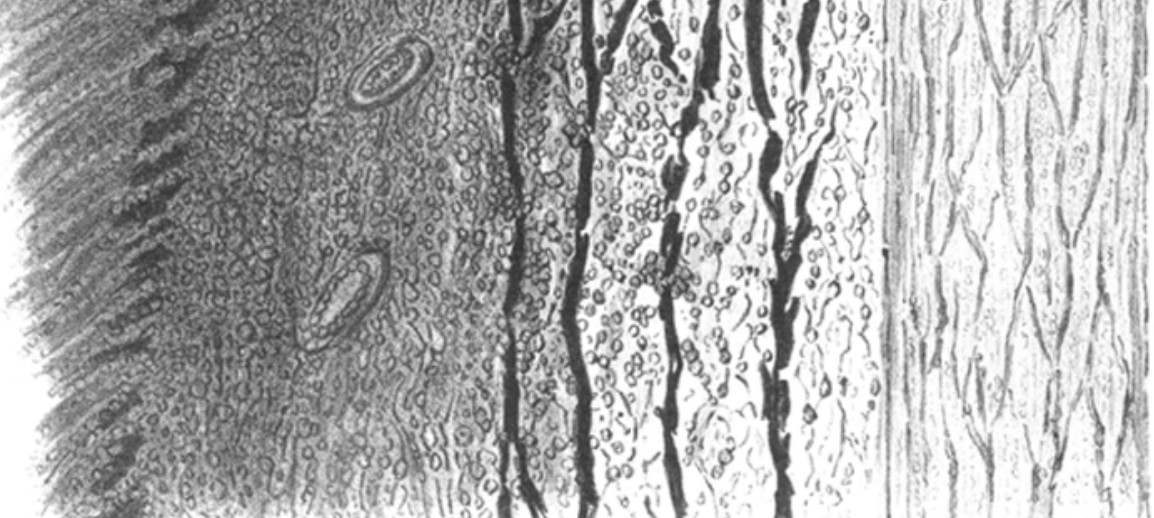

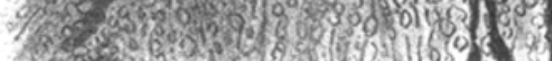
30 . 